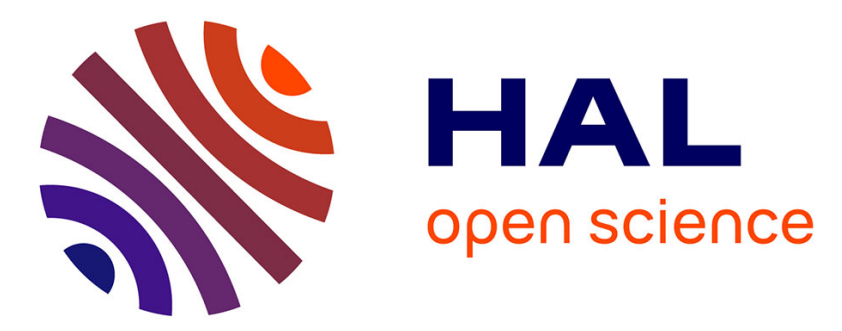

\title{
Compte-rendu bibliographique, Maïté Billoré, De gré ou de force. L'aristocratie normande et ses ducs (1150-1259)
}

\author{
Laurence Moal
}

\section{- To cite this version:}

Laurence Moal. Compte-rendu bibliographique, Maïté Billoré, De gré ou de force. L'aristocratie normande et ses ducs (1150-1259). Cahiers de Recherches Médiévales et Humanistes = Journal of Medieval and Humanistic Studies, 2014, 10.4000/crm.13346 . hal-03142750

\author{
HAL Id: hal-03142750 \\ https://hal.science/hal-03142750
}

Submitted on 16 Feb 2021

HAL is a multi-disciplinary open access archive for the deposit and dissemination of scientific research documents, whether they are published or not. The documents may come from teaching and research institutions in France or abroad, or from public or private research centers.
L'archive ouverte pluridisciplinaire HAL, est destinée au dépôt et à la diffusion de documents scientifiques de niveau recherche, publiés ou non, émanant des établissements d'enseignement et de recherche français ou étrangers, des laboratoires publics ou privés. 
Cahiers de recherches médiévales et humanistes

Journal of medieval and humanistic studies

Comptes-rendus | 2015

Maïté Billoré, De gré ou de force. L'aristocratie

normande et ses ducs (1150-1259)

\section{Laurence Moal}

\section{OpenEdition}

\section{Journals}

Electronic version

URL: http://journals.openedition.org/crm/13346

DOI: $10.4000 / \mathrm{crm} .13346$

ISSN: 2273-0893

\section{Publisher}

Classiques Garnier

\section{Electronic reference}

Laurence Moal, « Maïté Billoré, De gré ou de force. L'aristocratie normande et ses ducs (1750-1259)», Cahiers de recherches médiévales et humanistes [Online], Comptes-rendus, Online since 16 February 2015, connection on 15 October 2020. URL : http://journals.openedition.org/crm/13346 ; DOI : https:// doi.org/10.4000/crm. 13346

This text was automatically generated on 15 October 2020 .

(c) Cahiers de recherches médiévales et humanistes 


\title{
Maïté Billoré, De gré ou de force. $L$ 'aristocratie normande et ses ducs (1150-1259)
}

\author{
Laurence Moal
}

\section{REFERENCES}

Maïté Billoré, De gré ou de force. L'aristocratie normande et ses ducs (1150-1259), Rennes, Presses universitaires de Rennes, 2014, 448 p.

ISBN 978-2-7535-3328-8

1 Au cœur de l'Empire Plantagenêt, lieu de naissance de l'État moderne, disposant d'une documentation abondante, la Normandie des XII ${ }^{\mathrm{e}}$-XIII ${ }^{\mathrm{e}}$ siècles constitue un champ de recherche privilégié pour les médiévistes. Mais la noblesse normande n'avait pas encore fait l'objet d'une étude spécifique. Maïté Billoré a relevé le défi dans cet ouvrage qui est la version remaniée et allégée de sa thèse soutenue en 2005. Au cœur de son étude, sont développées les relations entretenues entre les nobles et le prince entre service et hostilité. Une réflexion approfondie est menée sur la nature oppressive et répressive du pouvoir (potestas) et la nature affective de l'autorité (auctoritas). M. Billoré a eu recours à des sources diverses, puisées dans les archives anglaises et normandes, en particulier des documents administratifs : actes de la pratique, lois et coutumiers, enquêtes, pièces de l'Échiquier, enrichis par une production historique, littéraire et politique de qualité.

Le livre se divise en sept chapitres. Les trois premiers traitent de la nature et du fonctionnement du groupe nobiliaire. M. Billoré commence par explorer l'identité individuelle du noble et la socio-genèse du groupe nobiliaire. Fruit d'un héritage composite, l'aristocratie normande se dote d'une identité collective qui se fonde sur la valorisation du sang. Les ancêtres font l'objet d'une véritable commémoration qui s'exprime dans les épitaphes des stèles funéraires, l'anthroponymie, la sigillographie, 
l'héraldique, autant d'instruments d'identification sociale qui participent à la constitution de la mémoire familiale. L'art funéraire, lieu de rencontre entre la vie et la mort, permet la consolidation des identités sociales. Ce thème est également développé dans la thèse de Xavier Storelli ${ }^{1}$, une référence pourtant absente de la bibliographie. On aurait d'ailleurs aimé pouvoir se référer à davantage de représentations iconographiques sur cet aspect, en particulier de gisants mentionnés dans l'étude, mais on ne trouve dans le livre qu'une photo de la dalle tumulaire de Raoul d'Aoust en 1269. La noblesse se signale encore dans les rapports sociaux qu'induit le fait de tenir un territoire et un pouvoir sur les hommes. À la base du dominium aristocratique, l'autorité et les redevances seigneuriales traduisent l'aspect personnel de la relation entre dominant et dominé.

3 Dès la fin du XII ${ }^{e}$ siècle, la socio-genèse de la noblesse est en marche. Le groupe aristocratique a acquis d'abord une existence «de fait». Puis, il prend conscience de son identité, résultat d'un processus de socialisation qui passe par le partage d'une activité commune, par l'adhésion à une éthique spécifique, ainsi que par l'adoption de codes comportementaux et culturels, comme la culture de l'apparence. L'aristocratie normande fonde également son identité sur la chevalerie et ses valeurs, exaltées très tôt dans les sources normandes. Entre 1180 et 1230, la fixation des statuts juridiques contribue à la transformation de l'aristocratie en noblesse de droit. Un tel processus est plus précoce qu'ailleurs du fait du dynamisme de l'administration ducale et des progrès de l'État naissant. En effet, la mise par écrit de la coutume, au début du XIII ${ }^{e}$ siècle, joue un rôle de catalyseur dans le processus d'identification sociale du groupe nobiliaire en statuant sur les règles qui s'appliquent à ses membres, alors que les vastes enquêtes entreprises par le duc contribuent à en mieux délimiter les contours.

Dans son deuxième chapitre, M. Billoré examine les pratiques familiales et les logiques de reproduction du pouvoir nobiliaire. La société aristocratique normande se révèle être un cas singulier dans la mesure où ses structures lignagères dominantes persistent bien que l'autorité du prince soit forte et que les guerres privées aient rapidement cessé. Le groupe nobiliaire déploie un certain nombre de tactiques afin de préserver l'unité du patrimoine familial, en prenant appui sur des dispositions légales édictées par le Très Ancien Coutumier. L'auteure analyse notamment les stratégies matrimoniales qui en découlent. Le champ géographique des alliances et les transferts patrimoniaux montrent que le mariage est un enjeu fondamental pour le lignage. Les femmes, bien qu'ayant un rôle peu favorisé sur le plan juridique, n'en sont pas moins des acteurs incontournables de la vie économique et politique car elles contribuent au maintien, voire à l'extension et donc à la renommée du lignage. On trouvera également un long développement sur l'ancrage territorial et castral qui se caractérise par des domaines patrimoniaux stables mais dispersés.

5 Le troisième chapitre se rapporte à la puissance, à la cohésion et à la hiérarchie du groupe nobiliaire. Il met en valeur l'importance et l'efficacité des réseaux familiaux, en particulier par ses manifestations économiques et sociales repérées dans les enquêtes permises par les cartulaires des abbayes du Tréport, de Mont-Morel et de la Lucerne. Les sources archivistiques permettent, en outre, de mesurer les solidarités familiales dans l'adversité, notamment lorsque des terres sont injustement perdues. La cohésion du groupe se manifeste encore dans le soutien apporté aux accusés. Elle se fonde sur des rapports sociaux complémentaires. Toutefois les liens personnels et réels établis par la féodalité sont à la base de l'organisation sociale et le ciment du groupe nobiliaire 
normand. En matière de pyramide sociale, une enquête de 1172 révèle trois niveaux : le duc, les tenants et les vassaux de ces derniers, qui, selon leur statut et à leur niveau de richesse différencié, sont pris dans des relations de dépendances qui structurent et solidarisent la société aristocratique. Pour déterminer le réseau féodal d'un seigneur, M. Billoré a recours aux listes de témoins et constate une grande stabilité de l'aristocratie locale sur un siècle. Elle examine encore avec soin les obligations du vassal ainsi que les liens très étroits entretenus avec le monde ecclésiastique. La pyramide est tenue et fortement contrôlée par le duc, ce qui constitue une spécificité normande. Aussi les nobles, dont le statut juridique est bien défini, sont-ils des soutiens et des relais du pouvoir.

6 Les trois derniers chapitres portent sur les bouleversements provoqués par l'affirmation de la royauté. En effet, dans la seconde moitié du XII ${ }^{e}$ siècle, les rois d'Angleterre se montrent très novateurs en matière d'idéologie du pouvoir et s'emploient à asseoir leur autorité aux dépens de leurs vassaux.

7 Le quatrième chapitre s'attache à montrer l'encadrement, le contrôle et l'oppression exercés par les Plantagenêts. La noblesse est alors plus étroitement surveillée et assujettie, mais quelques familles frondeuses osent pourtant affronter le pouvoir. Face à une aristocratie très ancrée territorialement, les Plantagenêts s'appliquent à occuper le terrain. C'est d'ailleurs une "géographie de l'insoumission» qui oriente les déplacements de la Cour. Le prince renforce sa présence par des voyages incessants, ce qui lui permet de mettre en ordre l'administration et de consolider ses liens avec les seigneurs locaux. Le contrôle du réseau castral est un enjeu majeur pour les Plantagenêts. Pour l'exercer, ils s'appuient sur un règlement contenu dans les Consuetudines et justiciae de 1091, législation réaffirmée par Henri II et qui n'autorise les constructions aristocratiques que tant qu'elles ne portent pas atteinte au pouvoir du duc. Profitant des règles du droit féodal, le duc peut encore confisquer, voire démanteler des forteresses. Il a recours à des oppressions fiscales envers tous ceux qui se rendent coupables de félonie. La mise au pas de l'aristocratie passe aussi par d'autres actions répressives qui peuvent concerner les héritages et les alliances matrimoniales. Le Statut of Treason définit le crime de trahison, concept particulièrement sensible dans un monde fondé sur des liens de fidélité d'homme à homme. Par ailleurs, les exigences royales en matière d'obligations militaires s'accroissent. Cette «étatisation de la violence " va à l'encontre des pratiques traditionnelles de l'aristocratie qui subit les dérives autocratiques du pouvoir. Néanmoins, le duc sait aussi avoir recours à des actions non-répressives qui consistent, par exemple, à récompenser les fidélités afin de prévenir les trahisons. M. Billoré précise qu'il n'y a pas de règle absolue qui explique l'emploi de tel ou tel type d'action.

8 Le cinquième chapitre traite de la curialisation et la "fonctionnarisation" de l'aristocratie. Les nobles participent au pouvoir, soit de manière informelle, soit au service du duc par la détention d'offices qui se multiplient et se hiérarchisent. L'aristocratie normande est très impliquée et bien représentée dans l'entourage ducal. L'administration demeure majoritairement aux mains des Normands, contrairement à ce qui se passe en Angleterre. Même si on note l'apparition d'hommes "nouveaux », la composition de la Cour évolue surtout au bénéfice des membres de la petite et moyenne noblesse sans toutefois que les Grands soient mis à l'écart. M. Billoré montre que les nobles ont contribué au développement d'un système administratif dont l'efficacité se révèle contraire à leurs intérêts. Les curiales se livrent entre eux à une véritable 
compétition. Par ailleurs, la forte participation des clercs et des prélats constitue une source de tensions entre Henri II et l'aristocratie locale. D'une manière générale, les Angevins tirent profit de la présence des nobles à la Cour afin de les neutraliser politiquement mais aussi pour mieux les soumettre à leur idéologie.

9 Dans le sixième chapitre, qui porte sur les chevaliers normands à l'épreuve de la guerre, M. Billoré explique comment se déroule le conflit qui engage le sort du duché de Normandie à l'époque de Philippe Auguste et quelle part y prennent les nobles. Après un développement sur les aspects militaires et juridiques de la conquête, elle s'interroge sur l'engagement militaire des Normands et le désastre de 1204. Dès avril 1193, les premières offensives déstabilisent l'aristocratie normande. Toutefois, sa motivation est à son comble avec le retour de Richard Cœur de Lion de 1194 à 1199. Qu'en est-il ensuite? Si le facteur humain joue un rôle essentiel dans la défaite, M. Billoré met en doute la lâcheté des défenseurs de Rouen en juin 1204. Elle évoque plutôt les difficultés d'une armée mal encadrée et qui n'exploite pas les possibilités stratégiques offertes par le réseau castral. Les sources pointent du doigt des failles dans l'organisation de la défense. Pour autant, qu'en est-il de la question de la trahison des Normands? Il y a bien des défections dans les zones frontalières, mais pareille attitude n'est pas inhabituelle à l'époque. Cependant, celle de Robert d'Alençon, fin 1202, racontée dans l'Histoire de Guillaume le Maréchal, constitue un tournant majeur, car il s'agit d'un seigneur possessionné en plein territoire angevin. C'est ailleurs cependant qu'il faut chercher pour comprendre les véritables raisons de la défaite. La situation de guerre longue motive le désir de paix chez l'aristocratie normande. Le désaveu de Jean sans Terre est également un fait important. Ce duc «étranger ", choisi par défaut, a une attitude éloignée du portrait idéal, toutes choses qui suscitent des sentiments hostiles, surtout après l'assassinat d'Arthur de Bretagne en 1199. Ce discrédit, qui affecte Jean sans Terre, fragilise le lien féodal qui rattachait les nobles normands à ce roi et explique leur ralliement au roi de France.

10 Le septième chapitre porte sur les réactions, les stratégies et le devenir du groupe aristocratique normand au lendemain de la conquête et de la chute de l'Empire Plantagenêt. Philippe Auguste exige des nobles normands plus qu'un hommage-lige. Il leur impose un engagement définitif et exclusif, afin de réaliser une rupture entre l'Angleterre et la Normandie. La notion antique de territorium est ressuscitée et, selon cette notion, à un espace ne doit plus correspondre qu'une seule autorité. De son côté, Jean sans Terre refuse également la double-allégeance: il n'est plus possible de posséder des fiefs de part et d'autre de la Manche. Dans un tel contexte, que deviennent les héritages? L'après-guerre et les tensions persistantes entre les rois de France et d'Angleterre sont propices aux confiscations et redistributions, ce qui déstabilise en profondeur l'aristocratie et met à l'épreuve la solidarité du groupe. Malgré un désir d'apaisement, le roi de France s'emploie à éliminer le niveau le plus élevé de la hiérarchie nobiliaire. Les nobles normands, quant à eux, mettent au point des stratégies familiales afin de conserver leurs domaines et contourner l'interdit concernant les doubles allégeances. Les partages, par exemple, témoignent de la capacité d'adaptation de ces familles, mais aussi de la volonté de s'affranchir du poids de la coutume.

11 Face à l'établissement du régime capétien, assiste-t-on à l'émergence d'une nouvelle société nobiliaire ? Selon le schéma établi par Lucien Musset et devenu classique, après 1204, la Normandie est décapité de sa classe dirigeante et bouleversée. Il est vrai que les barons disparaissent dans leur majorité. Mais l'établissement du gouvernement 
français est aussi un facteur de promotion sociale pour ceux qui restent. Une analyse fine montre que le monde des petits seigneurs est d'une grande stabilité et que, plus haut dans la hiérarchie, de nouveaux seigneurs apparaissent. Ils sont surtout français, installés par le roi à son retour de croisade. Ce sont eux les grands bénéficiaires des redistributions de terre. Cependant, pour M. Billoré, il est faux de prétendre que la noblesse française a envahi le duché. Le roi évite de provoquer l'indignation des seigneurs et préfère mener une politique " démagogique » tout en préservant les us et coutumes. Certes, il confie les postes clés aux seigneurs français mais il emploie localement des Normands, qui perpétuent l'efficacité de l'administration ducale. Le poids du joug français sur la société normande reste pourtant difficile à évaluer. Ponctuellement, on note des tentatives insurrectionnelles, des complots même contre le roi. Mais ce dernier affiche sa volonté de pacification qui se traduit par une intense propagande, élaborée par les intellectuels pour valoriser l'image du prince. Non seulement cette campagne de séduction compense l'action répressive, mais elle conduit aussi à la rupture définitive du lien affectif qui unit l'aristocratie normande et les Plantagenêts.

12 Avec cet ouvrage, il convient de saluer un essai très complet et intéressant d'anthropologie historique d'un groupe nobiliaire, où sont explorés la logique et l'imaginaire aristocratique selon une méthode phénoménologique. Même si M. Billoré n'a pas pu publier toutes les informations de sa thèse, on trouvera dans ce livre de nombreux sceaux nobiliaires, des photographies de mottes et châteaux, des cartes, ainsi qu'en annexes des tableaux de filiation reconstituant les familles et leurs alliances. En définitive, comme le signale Martin Aurell dans sa préface, M. Billoré nous livre un ouvrage très riche sur l'histoire sociale du Moyen Âge.

\section{NOTES}

1. Xavier Storelli, Le chevalier et la mort dans l'historiographie anglo-normande (XI siècle-début du XIII siècle),thèse de doctorat, Université de Poitiers, 2009. 